Mens

revue d'histoire intellectuelle de l'Amérique française

\title{
La bibliothèque du Conseil supérieur de Québec (1717-1760) : formation et contenu
}

\section{François Melançon}

Volume 5, numéro 2, printemps 2005

L'histoire du livre au Québec, de la Nouvelle-France au XX ${ }^{\mathrm{e}}$ siècle

URI : https://id.erudit.org/iderudit/1024357ar

DOI : https://doi.org/10.7202/1024357ar

Aller au sommaire du numéro

Éditeur(s)

Centre de recherche en civilisation canadienne-française

ISSN

1492-8647 (imprimé)

1927-9299 (numérique)

Découvrir la revue

Citer cet article

Melançon, F. (2005). La bibliothèque du Conseil supérieur de Québec (1717-1760) : formation et contenu. Mens, 5(2), 277-305.

https://doi.org/10.7202/1024357ar
Résumé de l'article

En Nouvelle-France, le livre joue un rôle de premier plan dans la rencontre entre l'institution juridique d'origine européenne et le " vivre ensemble " colonial modulé par un espace étranger, l’Amérique du Nord. Il constitue un support de l'arsenal législatif des autorités monarchiques et un support du droit coutumier parisien qui régit la vie civile de la colonie. C'est pourquoi l'acquisition des livres de judicature est devenue, tôt dans le XVIII ${ }^{\mathrm{e}}$ siècle, une question clé des débats relatifs à la qualité de l'administration judiciaire de la Nouvelle-France et aux compétences juridiques de ses officiers. Laissée traditionnellement à la charge des magistrats, cette démarche va peu à peu être assumée par le gouvernement qui verra à fournir un certain nombre de livres de référence à sa magistrature locale. La bibliothèque du Conseil supérieur de Québec deviendra ainsi la première bibliothèque de collectivité laïque dans la vallée laurentienne avant les années 1760. Le présent article en présente son histoire et son contenu ainsi qu'un catalogue reconstitué de la collection. 


\title{
LA BIBLIOTHÈQUE DU CONSEIL SUPÉRIEUR DE QUÉBEC (1717-1760) : FORMATION ET CONTENU.
}

\author{
François Melançon \\ Département de lettres et communication \\ Université de Sherbrooke \\ francois.melancon@usherbrooke.ca
}

\section{Résumé}

En Nouvelle-France, le livre joue un rôle de premier plan dans la rencontre entre l'institution juridique d'origine européenne et le « vivre ensemble » colonial modulé par un espace étranger, l'Amérique du Nord. Il constitue un support de l'arsenal législatif des autorités monarchiques et un support du droit coutumier parisien qui régit la vie civile de la colonie. C'est pourquoi l'acquisition des livres de judicature est devenue, tôt dans le XVIII' ${ }^{\mathrm{c}}$ siècle, une question clé des débats relatifs à la qualité de l'administration judiciaire de la Nouvelle-France et aux compétences juridiques de ses officiers. Laissée traditionnellement à la charge des magistrats, cette démarche va peu à peu être assumée par le gouvernement qui verra à fournir un certain nombre de livres de référence à sa magistrature locale. La bibliothèque du Conseil supérieur de Québec deviendra ainsi la première bibliothèque de collectivité laïque dans la vallée laurentienne avant les années 1760. Le présent article en présente son histoire et son contenu ainsi qu'un catalogue reconstitué de la collection.

\section{Abstract}

In New France, books played a key role in acquainting the colonial vivre ensemble with European judicial institutions. They buttressed the Custom of Paris, which governed civil affairs in the colony, and were a mainstay in the royal authority's legislative arsenal. Accordingly, in the early eighteenth century, the 
acquisition of books relating to judicature became a key issue in the wider debate surrounding New France's judicial administration and the legal aptitudes of its officials. Traditionally left in the hands of magistrates, this task was gradually assumed by the government which sought to make legal reference books more accessible to the local magistrature. As a result of this endeavour, Quebec's Superior Council library became the first secular special library in the St. Lawrence $V$ alley before the British conquest. This article outlines the library's history and provides the reader with a reconstructed catalogue of its collection.

L'écriture occupe une place centrale dans les systèmes juridiques occidentaux. Jack Goody a déjà souligné l'enracinement historique de cette osmose de l'écriture et de la loi ${ }^{1}$ qui a permis aux représentations graphiques de la parole de participer des manifestations de l'ordre, de son application et de son maintien. Depuis le $\mathrm{XVI}^{\mathrm{e}}$ siècle, la montée en force du droit écrit et du droit jurisprudentiel, la compilation des droits coutumiers et l'ascension de la valeur probatoire de l'écrit devant les tribunaux ont consolidé cette alliance ${ }^{2}$. Toute une littérature juridique a dès lors vu le jour : recueils de textes de lois et de décisions judiciaires, commentaires sur cette législation, traités théoriques et manuels de procédures ${ }^{3}$. Autant de partenaires obligés avec lesquels l'homme de loi a dû entreprendre des échanges et des dialogues virtuels. La qualité de ces dialogues avec les sources écrites, la maitrise des textes de lois pour hiérarchiser leur autorité et l'habileté intellectuelle à tirer d'un précédent le modèle capable de régler un cas particulier, inédit, allaient désormais prévaloir ${ }^{4}$.

L'accès aux livres revêt donc une importance particulière pour les membres de la magistrature. Ce sont des instruments de travail qui possèdent sur le plan juridique le même statut que les outils de l'artisan, c'est-à-dire qu'ils comptent parmi leurs propres au sein d'une communauté matrimoniale ${ }^{5}$. Ainsi, l'acquisition des livres de droit par les magistrats re- 
lève d'abord d'une responsabilité personnelle. Les institutions qui administrent la justice se chargeront de cette tâche plus tardivement. Dans le royaume de France, par exemple, les premières réalisations en ce sens font leur apparition au cours du dernier tiers du XVII ${ }^{e}$ siècle à la faveur de la création de bibliothèques destinées aux membres de diverses associations et de l'ouverture au public lettré de volumineuses collections privées ${ }^{6}$.

La situation diffère peu en milieu colonial. À Québec, capitale de la Nouvelle-France, il semble longtemps revenir aux différents officiers de justice d'acquérir leurs propres livres de droit, en dépit de ressources financières jugés modestes et d'un marché local du livre étriqué, dont l'offre dépasse rarement les prescriptions ecclésiastiques destinées aux écoliers et aux fidèles? ${ }^{7}$. Cependant, la volonté de donner au développement colonial des structures d'encadrement qui permettent l'établissement d'une population stable et l'organisation d'un "vivre ensemble " policé a conféré un rôle régulateur de premier rang au système judiciaire. L'efficacité et la célérité de ce système sont rapidement devenus une préoccupation des agents locaux du roi et l'accès des magistrats aux livres de droit, une des pistes de solution.

Le présent article s'intéresse à la constitution de la première bibliothèque de collectivité laïque connue dans la vallée laurentienne avant les années $1760:$ la bibliothèque du Conseil supérieur de Québec. Ancêtre en quelque sorte de la bibliothèque parlementaire québécoise étudiée précédemment avec brio par Gilles Gallichan ${ }^{8}$, on en connaissait l'existence par certaines allusions, mais elle n'avait jamais fait l'objet d'une étude spécifique". Et surtout, on en ignorait le contenu et l'histoire. C'est le double objectif de ce texte. Il veut dégager dans un premier temps les grandes lignes du développement de cette bibliothèque depuis les premières réclamations de l'intendant 
François de Beauharnois, en 1702, jusqu'aux dernières commandes connues de l'intendant François Bigot, en 1750, et les emprunts que le procureur général du Conseil, LouisGuillaume Verrier, y effectue à la toute fin du Régime français. Il entend, dans un second temps, reconstituer le catalogue de la collection de cette bibliothèque dans l'état où elle se trouvait à partir de la fin des années 1730 . Une telle étude permet ainsi de mettre en lumière le rôle joué par le circuit institutionnel gouvernemental dans la diffusion du livre et de la culture imprimée en Nouvelle-France ainsi que de fournir, du même coup, un instrument de travail pour les chercheurs qui s'intéressent à la culture juridique québécoise et aux fondements juridiques de cette colonie française nord-américaine.

\section{Le Conseil supérieur : bref rappel historique}

Le Conseil souverain de la Nouvelle-France nait en avril 1663 d'une décision royale. Louis XIV, qui venait de recouvrer à peine deux mois plus tôt la "propriété du paÿs ", estime l'établissement d'un tel tribunal essentiel à son essor, ainsi qu'au « repos » et à la « félicité » de ceux qui l'habitent ${ }^{10}$. Avant toute autre prérogative, selon la tradition monarchique, le roi de France est d'abord un roi justicier ${ }^{11}$. D'où ce soin particulier à «l'établissement de la justice » qu'il considère comme «le principe et le préalable absolument nécessaire pour bien administrer les affaires et assurer le gouvernement, dont la solidité dépend autant de la manutention des lois et de nos ordonnances, que de la force de nos armes ${ }^{12} \%$. L'établissement du Conseil souverain/supérieur apparaît d'autant plus pertinent que la distance qui sépare Paris et Versailles de Québec, siège de cette nouvelle cour supérieure, nuit à l'exercice d'une " justice réglée » et diligente. Il appartient donc à ce dispositif administratif et juridictionnel de «faire fleurir les lois, maintenir et appuyer les bons, châtier 
les méchants et contenir chacun dans son devoir ${ }^{13}$ ". L'initiative n'est ni neuve ni unique. Il s'en trouve dans plusieurs provinces réunies ou conquises situées en Europe dans les franges du royaume, dont l'Alsace (1657) et le Roussillon (1660), de même que dans les autres colonies françaises d'Amérique et d'Asie.

Les conseils souverains/supérieurs ne sont ni plus ni moins que des parlements ${ }^{14}$. Comme eux, ils disposent du droit de décision en matière judiciaire et d'intervention dans le processus législatif ou, pour reprendre les mots d'Élise Frêlon dans son étude récente sur les pouvoirs normatifs du Conseil supérieur de Québec, de participer «à l'élaboration et à la diffusion de la norme royale " ainsi qu'à la "création de la norme provinciale ${ }^{15} »$. Ils rendent la justice au nom du roi et leurs jugements sont de dernier ressort, bien que le roi puisse évoquer à sa guise une affaire en cours ou casser un arrêt rendu $^{16}$. Ils doivent aussi vérifier, enregistrer et publier les actes royaux et interviennent sur le plan législatif et réglementaire, par provision, dans l'étendue de leur juridiction sur des questions d'intérêt régional ou local.

Les conseils souverains/supérieurs reposent cependant sur un mode d'organisation simplifié en regard de celui des parlements ${ }^{17}$. Ils se composent d'un effectif nettement moindre et, dans le cas des colonies, plus varié et adapté aux particularités du gouvernement local. À Québec, à l'origine, les cinq magistrats partagent leurs prérogatives avec le gouverneur - représentant officiel du roi - et le premier ecclésiastique de la colonie - pour lors François de Laval —, au demeurant responsables de leur nomination. Devant eux, un procureur général siège, chargé de la défense des intérêts du roi, de la Couronne et de la société. La compagnie se complète d'un greffier et d'un huissier ${ }^{18}$. 
Le Conseil souverain/supérieur de Québec subit une première modification substantielle en 1675. Le nombre de conseillers est alors porté à sept - nommés désormais par le roi. L'intendant devient officiellement membre ex officio du tribunal, troisième en importance dans la hiérarchie des honneurs, après le gouverneur et l'évêque, mais coiffé du titre de "président». Cela lui assure un plus grand pouvoir effectif que le gouverneur dont le titre de "chef » du conseil est essentiellement honorifique. Dès lors, le conseil allait devenir le lieu institué de la « joute symbolique ${ }^{19}$ » qui opposera, avec plus ou moins de virulence selon la personnalité et le statut des personnes en titre, le gouverneur et l'intendant ${ }^{20}$.

En 1703, le nombre de conseillers est porté à douze, dont une charge est réservée à un ecclésiastique. Le conseil se compose désormais de dix-sept membres, auxquels deux assesseurs, qui n'ont qu'une voix consultative, s'ajoutent à partir de 1742. On souhaite en haut lieu que l'ouverture de ces deux postes suscite l'esprit d'émulation dans la colonie et attire de bons candidats à la magistrature. Pour cette raison, ils sont réservés aux étudiants des conférences de droit que le procureur général Louis-Guillaume Verrier donne une à deux fois par semaine, chez lui, depuis $1733^{21}$.

Le recrutement représente un problème chronique pour le Conseil supérieur en raison, allègue-t-on, de la faiblesse du traitement des conseillers. Ceux-ci reçoivent jusqu'au début du deuxième tiers du XVIII ${ }^{\mathrm{e}}$ siècle 300 livres par année, à l'exception du premier conseiller qui se voit attribuer 500 livres. En 1733, dans l'espoir de doper le recrutement et surtout le recrutement de candidats de qualité, les premier et deuxième conseillers en titre obtiennent à leur tour un traitement distinctif. Alors que les gages des conseillers ordinaires restent à 300 livres, ceux des trois premiers magistrats s'établissent désormais à 450 livres, niveau que leurs confrères n'atteindront que deux décennies plus tard (1752), au même moment 
où leurs propres gages seront pour leur part majorés à 600 livres.

La modicité du traitement aurait pu trouver une compensation dans les honneurs conférés à cette charge, mais ce n'est pas le cas en Nouvelle-France. Dans cette société d'Ancien Régime où la mise en représentation des particuliers joue un rôle significatif dans l'organisation et la hiérarchisation de la société civile, foin d'honneurs disproportionnés pour ces magistrats du bout du monde! Certes, ils peuvent assister en corps aux cérémonies publiques et devancer les marguilliers à l'église. Toutefois, sur le plan individuel, ils sont « de capa et de spada", selon le trait d'ironie du baron de Lahontan ${ }^{22}$, c'est-àdire qu'ils ne portent ni l'épée ni la robe, de quelque couleur que ce $\operatorname{soit}^{23}$. A en croire le militaire philosophe, ils se confondent avec les bourgeois de la ville. De plus, la charge n'anoblit pas dans la colonie. Enfin, puisque ni vénalité ni patrimonialité ne se pratiquent dans la colonie, les conseillers sont soumis au bon vouloir du roi qui peut les révoquer en tout temps.

\section{La bibliothèque du Conseil}

\section{La formation de la bibliothèque du Conseil}

La première allusion à la présence de livres au Conseil supérieur de Québec apparaît dès 1687, alors que Jean-Baptiste Colbert, marquis de Seigneley, secrétaire d'État à la Marine, expédie aux magistrats un Recueil des édits, déclarations et ordonnances concernant les duels et les rencontres ${ }^{24}$ qu'ils décident de garder au greffe "pour y avoir recours quant [sic] besoin $\operatorname{sera}^{25} »$. Cette allusion désigne déjà un type d'ouvrages, le recueil de lois, un champ d'expertise, le droit civil, et un « lieu » de conservation, le greffe ${ }^{26}$. La réception de tels actes royaux de juridiction générale ou particulière est régulière. Ces textes 
de lois sont lus, commentés et, lorsque nécessaire, diffusés à travers la colonie. Les allusions à leur conservation restent toutefois rares, comme si elle allait de soi, nous laissant ainsi perdus en conjectures face aux modalités de cette conservation $^{27}$.

L'intendant François de Beauharnois est le premier représentant du roi à marquer sa préoccupation envers le difficile accès aux livres de judicature. Issu d'une famille qui a une longue tradition d'études juridiques et doté d'une certaine érudition ${ }^{28}$, le nouvel intendant endosse le discours « misérabiliste » ambiant sur la condition financière des magistrats du Conseil. Il rapporte ainsi en 1702 au ministre de la Marine, son cousin par alliance, que ces hommes de lois sont incapables de s'acheter «les livres qui leur seraient nécessaires pour bien juger les différens procés » en raison de la modestie de leurs gages. Pour remédier à la situation, il propose de récupérer les gages des deux charges vacantes, soit une somme de 600 livres, pour l'appliquer à l'achat de livres de judicature. Une telle manœuvre administrative, précise-t-il, " nous mettra en Etat d'avoir quelques livres qui appartiendront au con[sei]l sur les quels les rapporteurs iront travailler l'ors qu'ils auront des procés a Instruire ${ }^{29} »$. Les autorités métropolitaines ne semblent pas acquiescer à cette proposition puisque Louis XIV ne se contente pas seulement de combler les deux sièges vacants, mais il procède de plus à l'augmentation du nombre de conseillers, qui passe en ce début de XVIII ${ }^{e}$ siècle de sept à douze.

L'administration monarchique ne parait donc pas partager les préoccupations de Beauharnois. Et la situation n'est pas en voie de s'améliorer s'il faut en croire les propos des procureurs généraux du Conseil. Dans une lettre que François-Madeleine Ruette d'Auteuil de Monceaux adresse aussi au ministre de la Marine, en 1704, il souligne que : 
Comme le Conseil Superieur est Remply presentement de Nouveaux Sujets qui nont Pas Seullement les Elemens de la pratique du droit n'y de la Coutume et qui ne sont plus daage ny En estat par leurs affaires d'y travailler Ils rendent des arrest qui y sont sy contraires que les moindres praticiens s'en Recrient ${ }^{30}$.

Son successeur, Mathieu-Benoît Collet, revient à la charge en 1714. Dans une récusation des critiques d'un conseiller sur sa façon de rendre ses conclusions d'enquête, il conclut sa missive au roi en précisant "qu'il n'y a point de Livres dans le Conseil quoy qu'il deut y en avoir suivant l'ordonnance ${ }^{31} »$. Il récidive trois ans plus tard avec plus de détermination. La question de la disponibilité de livres au Conseil s'inscrit cette fois dans un projet plus ambitieux et plus englobant. Profitant de son passage en France à l'automne 1716, afin de remettre personnellement une série de neuf mémoires destinés à améliorer l'administration de la justice en Nouvelle-France, il propose de donner des leçons de droit "pour que ceux qui aspirent aux charges de judicature puissent etre instruits ${ }^{32}$ ». La proposition plaît et semble réalisable. Il obtient à cet effet une somme de 500 livres afin d'acheter à Paris les livres nécessaires à cet enseignement ${ }^{33}$. Les ouvrages lui sont alloués à la condition qu'il donne gratuitement ses cours. Auquel cas, s'ils portent les fruits escomptés, Collet pourra se voir octroyer une gratification royale. La nature de l'entente fait en sorte que la garde des livres est confiée à l'intendant Michel Bégon. Il en va ainsi des livres du procureur comme des nouveaux instruments de mathématiques du jésuite responsable de leur enseignement au collège de Québec : ils demeurent la propriété du roi et ne sont que prêtés ${ }^{34}$. Annoncés en mars 1717, les livres arrivent à Québec au cours des mois suivants, alors que Collet ne rentre dans la colonie qu'à l'automne 1719. Par la suite, la correspondance officielle n'y fait plus référence, pas plus d'ailleurs qu'aux leçons de droit de Mathieu- 
Benoît Collet. Tout porte à croire que ces leçons n'ont jamais été données et que les ouvrages sont partis en fumée lors du deuxième incendie du palais de l'intendant en 1727.

Les modalités d'administration de la justice coloniale se transforment dans le tournant de la décennie 1720 à 1730 . D'abord, Gilles Hocquart, devenu officiellement intendant en 1731, réalise deux projets significatifs. D'une part, il procède à la construction d'un dépôt d'archives à l'épreuve du feu pour la conservation des archives coloniales (minutes notariales, registres du Conseil supérieur et de la Prévôté) ${ }^{35}$. D'autre part, il reprend l'idée de leçons de droit afin « de former dans le Pays méme quelques bons sujets dans la science du droit français ", dont il confie la tâche au nouveau procureur général du Conseil supérieur, Louis-Guillaume Verrier, arrivé dans la colonie en $1729^{36}$.

Puis, on observe un accroissement de la participation gouvernementale dans l'approvisionnement en livres des institutions judiciaires coloniales. Alors qu'en 1728, le gouverneur Charles de Beauharnois de la Boische et l'intendant par intérim François Clairambault d'Aigremont recommandaient l'expédition d'un plus grand nombre d'exemplaires des actes royaux pour en déposer au siège des trois gouvernements ${ }^{37}$, deux ans plus tard, le secrétaire d'État de la Marine, Jean Frédéric Phélypeaux, comte de Maurepas, petit neveu du comte de Pontchartrain, prend l'initiative d'envoyer cinq exemplaires de la Compilation des ordonnances de la guerre de Briquet ${ }^{38}$. Ils sont destinés spécifiquement au gouverneur général, à l'intendant, aux gouverneurs particuliers de Montréal et de TroisRivières, ainsi qu'à Pierre de Rigaud de Vaudreuil de Cavagnial, un protégé du ministre, pour lors aide-major dans les troupes de la Marine ${ }^{39}$.

Le noyau de la bibliothèque du Conseil supérieur arrivera dans la foulée, en 1732. Il se compose d'une vingtaine 
de livres de références au majestueux format in-folio. Le gouverneur de Beauharnois et l'intendant Hocquart en accuse réception dans une lettre au ministre de Maurepas: «les livres de judicature dont sa Majesté a bien voulu faire La dépense sont $[. .$.$] dans La Chambre du Conseil ». Ces ouvra-$ ges ne répondent cependant pas à tous les besoins locaux, notent les deux administrateurs, «Lon y a recours assez Souvent dans Les causes difficiles : mais il s'en faut beaucoup quils puissent servir à L'instruction de ceux qui voudroient s'appliquer a l'étude de La jurisprudence ${ }^{40} »$. Le procureur général renchérit l'année suivante. Selon lui, les livres du Conseil sont si peu nombreux et si peu variés pour être utiles à son enseignement qu'il s'est senti contraint d'acheter personnellement " plusieurs livres » à cet effet $\mathrm{t}^{41}$. Dans la même veine, l'intendant Hocquart avait déjà pris l'initiative de faire venir d'Europe en 1731 douze exemplaires des Institutions an droit françois de Gabriel Argou ${ }^{42}$ qu'il entendait distribuer aux juges des différentes juridictions de la colonie et en faire déposer un exemplaire au greffe de chacune d'entre elles ${ }^{43}$.

De nouveaux ouvrages, toujours de référence, vont grossir le noyau primaire de la bibliothèque. Une commande passée par l'intendant François Bigot, au début des années 1750, prévoit ainsi l'ajout de cinq nouveaux ouvrages : les ordonnances de 1681, 1689 et 1690, relatives à l'administration de la marine marchande et militaire ${ }^{44}$, ainsi que le Dictionnaire de la Marine et du Dictionnaire d'Antoine Furetière ${ }^{45}$.

Le mystère persiste quant au destin de cette collection de livres à la suite du changement d'allégeance de la colonie dans les années 1760. Elle a pu accompagner les archives coloniales françaises dans leur périple, à moins qu'elle n'ait été conservée par les nouveaux administrateurs comme référence ou démembrée et vendue à certains lettrés de la capitale. Ces hypothèses restent les plus probables pour le moment. Une veille intellectuelle doit être maintenue à ce sujet. 
La correspondance officielle des administrateurs britanniques et les travaux exploratoires des "Messieurs de Québec » et de François-Joseph Cugnet sur le droit civil de la Nouvelle-France recèlent peut-être à ce propos des allusions significatives. Ce sont là des pistes à suivre.

\section{Le catalogue}

Deux séries documentaires ont servi à dresser le catalogue de la bibliothèque du Conseil supérieur de Québec. Le croisement de leur contenu respectif a permis l'identification, pour la majorité des ouvrages, d'au moins une des éditions disponibles sur les rayons de la salle du Conseil.

La série de documents initiale est formée des inventaires annuels du magasin du roi à Québec dans lesquels la première mention des «livres de judicature servant au Conseil Superieur de Quebec » apparait en $1738^{46}$. Vingt titres y sont alors répertoriés. À l'exception du Traité des droits bonorifiques, un nain parmi les géants avec son format in-douze, il s'agit tous d'ouvrages in-folio dont la majorité compte plus d'un volume. En tout, la collection rapportée en 1738 se compose de 41 volumes.

L'année suivante et au cours des années 1741 et 1742, la liste ne connait aucun changement à l'exception de la variation du nombre de tomes des Arrests de Louet qui s'élève à trois en 1738, mais à deux lors des trois autres inventaires. Les similitudes sont telles qu'elles laissent supposer que ces trois inventaires ne se sont pas faits livre en main, mais que le plumitif s'est contenté de retranscrire de fois en fois la liste précédente. Par contre, un nouvel inventaire in-visu semble dressé en 1743 puisque l'ordre d'apparition des titres diffère sensiblement. Ce nouvel ordre prévaut également dans les inventaires de 1744 et 1749 , seules autres années où l' « inventaire général des munitions et marchandises qui res- 
tent aujourd'hui dans les magasins du roi à Québec », dressé sur ordre du garde-magasin, rapporte cette liste de livres ${ }^{47}$.

Les inventaires annuels du magasin du roi fournissent donc le titre des ouvrages ${ }^{48}$, le nom de leur auteur principal $^{49}$, le nombre d'exemplaires ${ }^{50}$, le nombre de tomes de chacun d'entre eux ${ }^{51}$, ainsi que leur format ${ }^{52}$.

L'autre série documentaire a permis de préciser encore mieux la composition de la bibliothèque du Conseil. Elle comprend l'inventaire après décès du procureur général LouisGuillaume Verrier et, surtout, une note qui s'y trouve jointe sur laquelle sont inscrites les références des différents ouvrages de la bibliothèque du Conseil qu'il a empruntés en 1748, 1756 et $1758^{53}$. Ces références sont fort instructives puisqu'elles complètent les informations bibliographiques de onze des vingt ouvrages mis à la disposition des magistrats de Québec. Elles spécifient en outre le lieu et l'année d'édition.

La reconstitution du catalogue de la bibliothèque du Conseil supérieur de Québec repose donc sur le croisement des informations de ces deux séries documentaires. Elle n'est vraisemblablement pas exhaustive. Il semble que les titres portés à l'inventaire du garde-magasin ne soient que ceux de la collection fondatrice, expédiée en 1732. L'état des «balance et recette " des magasins du roi de Louisbourg, dressé en $1749^{54}$, et la commande de livres que François Bigot place en 1750 le suggèrent.

Afin de faciliter la poursuite de recherches ultérieures, nous avons pris soin d'indiquer la localisation des exemplaires correspondant, le plus souvent possible, aux éditions mises à la disposition des magistrats du Conseil.

\section{$* * *$}

Le livre joue un rôle de premier plan dans la rencontre entre l'institution juridique d'origine européenne et le « vivre 
ensemble » colonial modulé par un espace étranger, l'Amérique du Nord. Il constitue un support du droit coutumier parisien, que Louis XIV a élevé au rang de cadre juridique fondamental de la vie civile de la colonie en 1663, et de l'arsenal législatif des autorités monarchiques. Il sert aussi de véhicule aux précédents juridiques, sur lesquels va s'ériger peu à peu une jurisprudence locale, ainsi qu'aux interprétations attendues des différents codes de lois et aux procédures légitimes pour l'administration de la justice.

Laissée à la charge des magistrats, l'acquisition des livres de judicature est devenue, tôt dans le XVIII ${ }^{\mathrm{e}}$ siècle, une question clé des débats relatifs à la qualité de l'administration judiciaire de la Nouvelle-France et aux compétences juridiques de ses officiers. Les procureurs généraux du Conseil supérieur ont l'un après l'autre soulevé le point. Après l'envoi en 1717 d'une première collection de livres, peu documentée, vraisemblablement partie en fumée avec le palais de l'intendant dix ans plus tard, les autorités monarchiques semblent convenir du bien fondé des requêtes des administrateurs coloniaux. L'expédition d'un plus grand nombre d'exemplaires d'imprimés juridiques et le rétablissement d'une nouvelle bibliothèque à l'usage des membres du Conseil en témoignent.

Le gouvernement en vient donc à s'impliquer activement dans la diffusion de l'imprimé sur le territoire colonial, au côté des marchands détaillants et des établissements religieux. Si, à première vue, le circuit de diffusion qu'il anime ne bénéficie qu'à une classe déjà privilégiée de lettrés, son intervention trahit les liens plus profonds et maintes fois relevées par l'historiographie ${ }^{55}$ que l'État et le livre entretiennent, à l'exemple de la loi et de l'écrit. 


\section{Catalogue reconstitué de la BIBLIOTHÈQUE DU CONSEIL SUPÉRIEUR DE QuÉBEC}

\section{Sigles de localisation}

$\begin{array}{ll}\text { AEU } & \text { University of Alberta, Cameron Library } \\ \text { BAGB } & \text { Bibliothèque, Abbé Grégroire, Blois, France } \\ B m A & \text { Bibliothèque municipale d'Angers, France } \\ B m T & \text { Bibliothèque municipale de Troyes, France } \\ B N F & \text { Bibliothèque nationale de France } \\ B V A U & \text { University of British Columbia, Walter C. } \\ & \text { Koerner Library } \\ \text { NSHDL } & \text { Dalhousie University, Sir James Dunn Law } \\ & \text { Library } \\ \text { OGU } & \text { University of Guelph, McLaughlin Library } \\ \text { OHM } & \text { McMaster University, Mills Memorial Library } \\ \text { OKQJM } & \text { Queen's University, Douglas Library, W.D. } \\ & \text { Jordan Special Collections and Music Library } \\ \text { OKQL } & \text { Queen's University, William R. Lederman Law } \\ & \text { Library } \\ \text { OONL } & \text { Bibliothèque et Archives Canada } \\ \text { OOP } & \text { Bibliothèque du Parlement } \\ \text { OOU } & \text { Université d'Ottawa, Bibliothèque Morisset } \\ \text { OONMC } & \text { Musée canadien de la guerre, Bibliothèque } \\ & \text { Hartland Molson } \\ \text { OOSC } & \text { Cour suprême du Canada, Bibliothèque }\end{array}$


OSUL Universtié Laurentienne, Bibliothèque J.N.

Desmarais

OTU University of Toronto, Jobn P. Robarts Research

Library (Humanities and Social Sciences)

OTYL York University, Law Library

OWTU University of Waterloo, Library

QMM

McGill University, Humanities and Social Sciences

Library (McLennan/Redpath)

QMMRB MoGill University, Rare Books and Special

Collections Division, Library

QMML McGill University, Nabum Gelber Law Library

QMU Université de Montréal, Direction des bibliothèques

QQL Assemblée nationale du Québec, Bibliothèque

QQLA Université Laval, Bibliothèque générale

QSHERU Université de Sherbrooke, Bibliothèque des sciences bumaines

QSHERUD Université de Sherbrooke, Bibliothèque de droit et des publications gouvernementales

1. $\quad+$ Aubin, Nicolas ${ }^{56}$. Dictionnaire de Marine : contenant les termes de la navigation et de l'architecture navale. Amsterdam, J. Covens et C. Mortier, 1736. In $-4^{\circ}$.

Copies localisées $^{57}$ : BNF

2. + Argou, Gabriel (1640-1730). Institution an droit

francois. Paris, Jean Mariette, 1730. 2 v. $17 \mathrm{~cm}$.

Copies localisées : OONL; QMML; QMU ; QQLA.

3. Bacquet, Jean (-1597). Oeuvres de M. Jean

Bacquet...augmentees de plusieurs questions, decisions et arrests des cours souveraines de France. Paris, Thierry, 1688. 1063 p. 38 $\mathrm{cm}$.

Mentions documentaires ${ }^{58}$ : Oeuvres de Bacquet $\left(f^{\circ}\right) /$ 
Oeuvres de Bacquet ( $\mathrm{f}^{\circ}$, Paris 1688 ) Copies localisées: QQLA; NSHDL.

4. Bourdot de Richebourg, Charles A. Nouveau coutumier general: ou Corps des coutumes generales et particulieres de France, et des provinces connues sous le nom des Gaules...avec les notes de Toussaint Chawvelin, Julien Brodeau, \& Jean-Marie Ricard...jointes aux annotations de Charles du Molin, Francois Raguean \& Gabriel-Michel de la Rochemaillet. Mis en ordre, \& accompagne de sommaires en marge des articles...et enrichi de nowvelles notes. Paris, T. Le Gras, 1724. 4 v.; $38 \mathrm{~cm}$.

Mentions documentaires: Coutumier général $\left(\mathrm{f}^{\circ}\right)$ Copies localisées : NSHDL; OHM ; OONL; OOSC; QMML; QMU ; QQLA ; QSHERUD ; OSUL.

5. + Briquet, Pierre de. Code militaire; ou, Compilation des ordonnances des roys de France concernant les gens de guerre. Paris, Imprimerie royale, 1728.3 v. ; $17 \mathrm{~cm}$.

Copies localisées: OONMC

6. Brodeau, Julien (-1635). Covstume de la prevoste et vicomte de Paris. Paris, Guignard, I., 1669. 2 v., $37 \mathrm{~cm}$. Mentions documentaires : Coutume $\left(\mathrm{f}^{\circ}\right)$, par Brodeau / Coutume de Paris, par Brodeau (2 v. fo, Paris, 1669) / Commentaire sur la coutume de Paris, par Brodeau (2 v., $\mathrm{f}^{\mathrm{D}}$,

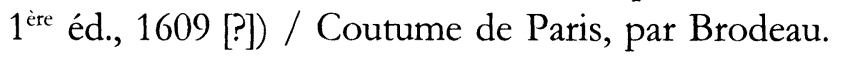
Copies localisées : OOU ; QMU ; QMML ; QQL ; QQLA. 7. + Choppin, René (1537-1606). Trois livres du domaine de la Couronne de France. Paris, E. Richer, 1634. 1 v. (320 p.), $\mathrm{f}^{\mathrm{o}}$.

Mentions documentaires : (1 v., 1634) / de Copin (Paris, 1639).

Copies localisées: (Médiathèque FM-Poitiers) A 1460, Fonds ancien. 
8. Coquille, Guy (1523-1603). Oenures de maistre Guy Coquille, sieur de Romenay: contenant plusieurs traitez touchant les libertez de l'Eglise gallicane, l'bistoire de France \& le droit francois. Bordeaux, Claude Labottière, 1703. 2 v. ; $39 \mathrm{~cm}$.

Mentions documentaires: Des successions $\left(\mathrm{f}^{\mathrm{f}}\right)$, par Coquille / Oeuvres de Coquille (2v. en 1, $\mathrm{f}^{\circ}$, Bordeaux, 1700) /

Oeuvres de Guy Coquille (2 v. en 1, $\mathrm{f}^{\circ}$, Bordeaux, 1703) / Oeuvres de Coquille.

Copies localisées: OHM ; OOSC ; OTYL; QMML; QMU ; QQL.

9. Du Perray, Michel (c.1640-1730). Traite des droits bonorifiques et utiles des patrons et curez primitifs, de leurs charges \& de celles des decimateurs. Paris, Paulus-du-Mesnils, 1733. [16], 587, [21] p. ;17 cm, $12 \mathrm{mo}$.

Mentions documentaires : Traité des droits honorifiques (12mo)

Copies localisées: QQQLA.

10. Dumoulin, Charles (1500-1566). Grand coutumier général, contenant toutes les coutumes généralles \& particulières $d u$ royaume de France \& des Gaulles; mesmement toutes celles qui ont esté redigees par les trois estats, \& homologuees, corrigees \& annotees de plusieurs decisions \& arrests, diligemment \& fidellement par messire Charles du Molin,...Premier tome. Paris, Jacques du Puys, 1567. [7, 1 bl.],CCCCCLI f. ; fo. Mentions documentaires : Arrêts de Dumoulin $\left(\mathrm{f}^{\mathrm{p}}\right)$. Copies localisées: Centre Toulousain d'histoire du droit et des idées politiques. Bibliothèque. Toulouse.

11. Ferriere, Claude de (1639-1715). Corps et compilation de tous les commentateurs anciens et modernes sur la coutume de Paris. Paris, Guignard, 1714. 4 v.; $40 \mathrm{~cm}$.

Mentions documentaires : Coutume de Paris $\left(\mathrm{f}^{\mathrm{p}}\right)$, par Ferrière / Coutume de Paris ( $f^{\mathfrak{p}}$, Paris, 1714) / Grand 
coutumier de Ferrière (4 v., fo, 1714) / Grand coutumier de Ferrière.

Copies localisées : NSHDL; OGU ; OONL; OOP ; OOSC ; OOU ; OSUL ; OTU ; OTYL ; OWTU ; QMBN ; QMML; QMU ; QQL ; QQLA ; QSHERU ; QSHERUD.

12. Fevret, Charles (1583-1661). Traite de l'abus, et du vrai sujet des appellations qualifiees du nom d'abus. Lyon, Girin et Riviere, 1677. $2 \mathrm{v}$.

Mentions documentaires: Traité de l'abus $\left(\mathrm{f}^{\circ}\right) /$ Traité de l'abus, par Fevret (2 vol. en 1, $\mathrm{f}^{\circ}$ ).

Copies localisées : OSUL

13. Furetiere, Antoine (1619-1688) ${ }^{59}$. Dictionnaire universel, contenant generalement tout les mots francois, tant vieux que modernes, \& les termes des sciences \& des arts ... Le tout extrait des plus excellens auteurs anciens et modernes. Ensuite corrige \& augmente par m. Basnage de Beauval, et en cette nouvelle edition, revu, corrige, \& considerablement augmente par $m$. Brutel de la Riviere. La Haye, P. Husson, 1727. 4 v.; $38 \mathrm{~cm}$. Copies localisées : QMBN ; QMU ; QQLA ; BUAU

14. Guenois, Pierre (1520-c1600) ${ }^{60}$. La grande conference des ordonnances et edicts royau jusques a l'annee 1659 / distribuee en 12 livres a l'imitation et selon l'ordre et disposition du Code de l'Empereur Justinianx. Paris, Guignard, 1692. 3 v.; in- $\mathrm{f}^{\circ}$. Mentions documentaires : Grande conférence des ordonnances $\left(f^{\circ}\right)$

Copies localisées : $\mathrm{BmT}$

15. Henrys, Claude (1615-1662). Recveil d'arrests remarquables, donnez en la cour de Parlement de Paris : pour la decision des plus notables \& singulieres questions ... Diuise en 6 liures. Paris, I. d'Alin, 1662-. $39 \mathrm{~cm}$.

Mentions documentaires : Arrêts de Henry (fo) / Arrêts de 
Henry (fo, 1 v. : $3 e$ ed, $1662 ; 2$ vol., 1660)

Copies localisées: QMML; QMU.

16. Le Brun, Denis (1640-1706). Traite des successions divise en quatre livres. Paris, s.n., 1700. [12], 735 p.

Mentions documentaires: Traité des successions $\left(\mathrm{f}^{\mathrm{D}}\right)$, par Le Brun / Traité des successions de Lebrun ( $f^{\circ}$, Paris $3 e$ éd., 1700).

Copies localisées :

17. Le Prestre, Claude. Questions notables de Droict décidées par plusieurs arrests de la cour de Parlement,... et divisées en 3 centuries... Paris, veuve G. Alliot, 1663. 992 p., f $\mathrm{f}^{\circ}$. Mentions documentaires: Oeuvres de Le Prestre $\left(\mathfrak{f}^{\circ}\right) /$ Arrêts de Le Prestre ( $f^{\mathfrak{p}}$, Paris, 1663).

Copies localisées : BmA

18. Louet, Georges (1540-1608). Recueil de plusieurs arrests notables du Parlement de Paris / pris des memoires de ... George Louet ... par ... Julien Brodeau. Paris, s.n., 1712. 2 v., 41 $\mathrm{cm}$.

Mentions documentaires : Arrêts de Louet $\left(f^{\circ}\right) /$ Arrêts de Louet (2 v., fo, Paris, 1712)

Copies localisées: QMML; QMU.

19. + Louis XIV. Ordonnance de Louis XIV roy de France et de Navarre: donnee a Fontainebleau an mois d'aoust 1681 touchant la marine. Paris, Denys Thierry et Christophile Ballard, 1681. [8], 273, [55] p. ; $25 \mathrm{~cm}$, in $-4^{\circ}$ Copies localisées: OONL, OTU, QQL.

20. + Louis XIV. Ordonnance de Louis XIV pour les armees navales et arcenaux de marine. Paris, Etienne Michallet, 1689. 407 p. ; $25 \mathrm{~cm}$. [In- $\left.4^{\circ}\right]$. Copies localisées : AEU, OKQJM, QQL. 
21. + Louis XIV.

Mentions documentaires : Ordonnance de 1690

22. Loyseau, Charles (1564-1627). Oenvres: contenant les cinq livres $d u$ droit des offices, les traitez des seigneuries, des ordres et simples dignitez, du deguerpissement et delaissement par hypotheque de la garantie des rentes et des abus des justices de village / suivant la correction et augmentation de l'auteur. Paris, C. de Sercy, 1678. 6 parties en 1 v., $39 \mathrm{~cm}$.

Mentions documentaires : Oeuvres de Loyseau $\left(\mathrm{f}^{\mathrm{0}}\right)$ /

Oeuvre de Loyseau $\left(\mathrm{f}^{\mathfrak{0}}\right.$, Paris, 1678)

Copies localisées: OOU; QMML.

23. Neron, Pierre. Les Edicts et ordonnances des tres cbrestiens roys, Francois I, Henry II, Francois II, Charles IX, Henry III, Henry IV, Louys XIII et Louys XIV, sur le fait de la justice et abreviation des procez, avec annotations, apostilles et conferences sous chacun article. Paris, Loyson, 1667. 1096 p., fo.

Mentions documentaires : Édits et ordonnances de Néron $\left(\mathrm{f}^{\mathrm{O}}\right)$

Copies localisées: QMBN.

24. Renusson, Philippe de (1632-1669). Traite de la subrogation de ceux qui succedent au lien et place des creanciers, on sont traittees les questions ardues et difficiles de cette matiere / par M. Ph. D. R. Paris, Le Gras, 1685. [viii], 525, [19] p.

Mentions documentaires: Traité de la subrogation $\left(\mathfrak{f}^{\circ}\right)$ Copies localisées: QMML.

25. Renusson, Philippe de (1632-1669). Traité des propres réels, réputés réels et conventionnels; où sont traitées les notables questions du droit Français divisé en 6 chapitres par P. H. D. R. Paris, Nicolas Le Gras, 1681. fo.

Mentions documentaires: Traité des propres $\left(\mathrm{f}^{\circ}\right)$

Copies localisées : BAGB. 
26. Ricard, Jean-Marie (1622-1678). Traite des donations entre-vifs et testamentaires. Paris, Michel Guignard et Claude Robustel, 1713. 2 v., $37 \mathrm{~cm}$.

Mentions documentaires: Des donations ( $\left.\mathrm{f}^{\circ}\right)$, par Ricard / Traité des donations par Ricard (1er vol., fo, 1713) / Traité des donations par Ricard (1er vol., $\mathrm{f}^{\circ}, 1713$ ) / Traité des donations par Ricard (1er vol., fo, 1713). Copies localisées: QMBN; QQLA; OOSC.

27. Mentions documentaires : Corps civil $\left(\mathrm{f}^{\mathfrak{f}}\right)^{61}$. 28. Mentions documentaires : Édits sur les reglements de la justice $\left(f^{0}\right)^{62}$.

\section{NOTES}

${ }^{1}$ Jack Goody, La raison graphique. La domestication de la pensée sawvage, Paris, Les éditions de Minuit, 1977, 275 p. ; La logique de l'écriture. Aux origines des sociétés bumaines, Paris, Armand Colin, 1986, pp. 133-169.

${ }^{2}$ Il serait évidemment naif d'évincer pour autant l'oral et le geste de la dynamique juridique. Ils restent des éléments clés, notamment dans l'administration de la justice, tant dans le discours que dans la pratique.

${ }^{3}$ Serge Dauchy, Synthèse du rapport : Les recueils d'arrêts et dictionnaires de jurisprudence, $X V T^{e}$-XVIII siècles, Centre d'histoire judiciaire, CNRS-Lille II, octobre 2002, 13 p.

${ }^{4}$ Thomas Würtenberger, "L'histoire des bibliothèques des juristes comme élément d'une histoire du droit et du savoir ", Sources. Travaux bistoriques, nos 41-42 (1995), p. 89.

${ }^{5}$ Thomas Würtenberger, loc. cit., pp. 89-101 ; François Bluche, Les magistrats du parlement de Paris au XVIII siècle (1715-1771), Paris, Les Belles Lettres, 1960, pp. 289-296. John A. Dickinson s'était déjà penché sur les bibliothèques de trois officiers de justice de la Nouvelle-France dans «Un aperçu de la vie culturelle en Nouvelle-France : l'examen de trois bibliothèques privées ", Revue de l'Université d'Ottawa, vol. 44, $\mathrm{n}^{\circ} 4$ (1974), pp. 453-466, tandis que la biographie que Cameron Nish a consacrée à l'entrepreneur et magistrat François-Étienne Cugnet comportait un chapitre complet sur ses livres : « L'image 
de l'homme ", dans François-Etienne Cugnet, 1719-1751 : entrepreneur et entreprises en Nouvelle-France, Montréal, Fides, 1975, pp. 145-156 et 164. Dans la même perspective, soulignons également les études de Jean-Claude Dubé sur les intendants de la Nouvelle-France : «Un milieu instruit et cultivé », dans Les Intendants en Nowvelle-France, Montréal, Fides, 1984, pp. 199-220 ; « La bibliothèque de Dupuy ", dans Claude-Thomas Dupuy, intendant de la Nouvelle-France, 1678-1738, Montréal/Paris, Fides, 1969, pp. 317-338 et 361-374.

"Pour un tableau de la situation française, voir Agnès Marcetteau-Paul, « Paris sous l'Ancien Régime », dans Claude Jolly, dir. Histoire des bibliothèques françaises, Paris, Promodis, 1988, t. II, pp. 428-434. Les véritables bibliothèques législatives prennent leur essor quant à elles dans le dernier quart du XVIII ${ }^{c}$ siècle. Gilles Gallichan évoque la création de « state library » à Montpellier (Vermont) et à Trenton (New Jersey) en 1777 et 1796, ainsi que la création de la « bibliothèque des députés » de l'Assemblée nationale française, en 1796, et celle du Congrès américain en 1800. Pour leur part, la Chambre d'assemblée et le Conseil législatif du Bas-Canada se sont dotés d'une bibliothèque destinée à leurs membres respectifs, en 1802. Voir Gilles Gallichan, Livre etpolitique au Bas-Canada, Sillery, Septentrion, 1991, p. 221.

${ }^{7}$ François Melançon, Le livre à Québec dans le premier XVIII siècle. La migration d'un objet culturel, thèse de doctorat (études françaises), Université de Sherbrooke, en cours.

${ }^{8}$ Gilles Gallichan, op. cit.

"Jean-Edmond Roy affirmait qu'il «il y avait dans les salles du Conseil supérieur une belle collection d'auteurs, propriété de l'État"; dans JeanEdmond Roy, L'ancien barreau au Canada, Montréal, C. Théoret, 1897, p. 25.

1" "Édit de création du Conseil souverain de Québec », avril 1663, dans Édits, ordonnances royaux, déclarations et arrêts $d u$ Conseil du roi, concernant le Canada, vol. 1:1627-1756, Québec, P.E. Desbarats, 1803, pp. 37 ss.

${ }^{11}$ Jean Barbey, « Droits régaliens », dans Lucien Bély, dir., Dictionnaire de l' Ancien régime, Paris, PUF, 1996, pp. 445-448.

${ }^{12}$ «Édit de création du Conseil souverain de Québec », avril 1663, ibid.

${ }^{13} \mathrm{Idem}$.

${ }^{14}$ Il faut garder à l'esprit que les parlements français d'Ancien Régime sont des cours de justice souveraines qui détiennent des compétences judiciaires, administratives et politiques qui ne doivent pas être confondues avec celles de 
l'institution politique délibérante, investie d'un pouvoir législatif, qui s'implante au Canada à partir de 1792 selon la tradition britannique.

${ }^{15}$ Élise Frêlon, Les pouvoirs du Conseil souverain de la Nouvelle-France dans l'édiction de la norme (1663-1760), Paris, L'Harmattan, 2002, 235 p.

${ }^{16}$ C'est la contradiction flagrante entre la « souveraineté » de ces cours et les prérogatives monarchiques de « retenus » qui sera à l'origine du changement de désignation de ces tribunaux de " conseils souverains " à « conseils supérieurs "; Bernard Barbiche, loc. cit., p. 362.

${ }^{17}$ Sur le fonctionnement des conseils et des parlements voir : Bernard Barbiche, "Cours souveraines ou supérieures », dans Lucien Bély, dir., Dictionnaire de l'Ancien régime, Paris, PUF, 1996, pp. 362-364 ; Olivier Chaline, «Parlements », ibid., pp. 960-965 ; Jean-Louis Harouel, « Conseils souverains », ibid., pp. 325-326 ; Jean-Louis Harouel, « Parlements », dans François Bluche, dir. Dictionnaire du Grand siecle, Paris, Fayard, 1990, p. 1153.

${ }^{18}$ Deux études d'ensemble dressent un tableau du Conseil supérieur de Québec et de ses développements. Il s'agit de R. Cahall, The Sovereign Council of New France, New York, 1915, 274 p. ; Jean Delalande, Le Conseil sowverain de la Nouvelle-France, Québec, Louis-A. Proulx, 1927, 358 p.

${ }^{19}$ Martin Pâquet, «Le "sport de la politique". Transferts et adaptations de la culture politique britannique au Québec, 1791-1960», dans Hélène Destrempes, Denise Meckle, Jean Morency et Martin Pâquet, dir., Des cultures en contact : visions de l'Amérique du Nord francophone, Québec, Nota Bene, 2005, pp. 149-171.

${ }^{20}$ L'évêque, pour sa part, devait voir décliner son pouvoir politique en proportion du discrédit qu'il avait acquis à la cour du roi et de ses absences répétées à son siège épiscopal. Aucune étude publiée n'a encore mis en lumière systématiquement cette " joute symbolique » entre gouverneur et intendant. Cependant, le livre de Christophe Horguelin, La prétendue république. Pouvoir et société au Canada (1645-1675) en montre très clairement les prémisses et la complexité des enjeux (Sillery, Septentrion, 1997).

${ }^{21}$ Le roi écrit à ce sujet à ses représentants locaux : « La preference [...] que Sa Ma.té veut bien donner aux sujets qui s'appliqueront a l'Etude du droit, pour les places de judicature [...] doivent exciter l'ambition et l'Emulation des jeunes gens de famille qui peuvent avoir des dispositions pour cette etude ; Et les S.rs de Beauh. Et hocq. Ne doivent rien negliger pour les engager a se rendre dignes de ces avantages et les porter pour ces effes aprofiter des Leçons que le Procureur general doit continuer de donner »; Mémoire du roi à Char- 
les de Beauharnois et Gilles Hocquart, 30 avril 1742, Bibliothèque et Archives Canada (BAC), Série $C^{11} A$, vol. 125, fol. 148v.

${ }^{22}$ Louis-Armand de Lom d'Arce, baron de Lahontan, Nouveaux voyages de Mr. le baron de Labontan dans l'Amérique septentrionale, dans Réal Ouellet, dir., Eurres complètes, Montréal, PUM, 1990, p. 273. Le militaire précise lui-même plus loin la signification de l'expression dans une « explication de quelques termes qui se trouvent dans le premier tome » : « c'est un titre de Gascogne que les gens de cette Province donerent autrefois par ironie aux Conseillers du Conseil Souverain de Canada, parce que les premiers Membres de ce Tribunal ne portoient ni robe, ni épée, se contentant de marche la canne à la main dans la Ville de Quebec, \& d'aller au Palais en cet équipage Bourgeois »; ibid., p. 513.

${ }^{23}$ Ailleurs dans le royaume, les conseillers des cours souveraines/supérieures siègent généralement en robe noire et assistent aux cérémonies publiques en robe rouge.

${ }^{24}$ Recueil des édits, déclarations, arrests et autres pièces concernant les duels et les rencontres, Paris, Sébastien Mabre-Cramoisy, 1669, viii-402 p. In-12.

${ }^{25}$ L'une des raisons invoquées pour garder l'ouvrage au greffe est la présence des autres pièces que l'ouvrage contient ; « délibérations du Conseil souverain ", 21 juillet 1687, dans Chronica I-Jugements et délibérations du Conseil souverain, 1663-1716 [CD-ROM], Montréal, Archives nationales du Québec/Société de la recherche Archiv-Histo, 1993, 1996 (1 disque).

${ }^{26}$ En fait, bien plus un responsable : le greffier.

${ }^{27}$ De toute façon, ces lois du royaume ou de la colonie, sous forme de compilations ou de feuillets individuels, ne semblent pas aux yeux des membres du Conseil être considérées comme des livres rattachés à leur pratique juridique.

${ }^{28}$ Jean-Claude Dubé, «François de Beauharnois de la Chaussaye », Dictionnaire biographique du Canada, Québec, PUL, 1974, vol. III, pp. 54-59.

${ }^{29}$ Lettre de François de Beauharnois à Jérôme Phélypeaux, comte de Pontchartrain, 11 novembre 1702, BAC, Série $C^{11}$ A, vol. 20, fol. 198v-199r.

${ }^{30}$ Lettre de François-Madeleine-Fortuné Ruette d'Auteuil de Monceaux à Jérôme Phélypeaux, comte de Pontchartrain, 14 novembre 1704, BAC, Série $\mathrm{C}^{11} \mathrm{~A}$, vol. 22, fol. 119-199v.

${ }^{31}$ Lettre de Mathieu-Benoît Collet à Louis XIV, 14 novembre 1714, BAC, Série $\mathrm{C}^{11} \mathrm{~A}$, vol. 34, fol. 338. À la lecture de l'ordonnance de 1667, il n'y a aucune référence à l'obligation de conserver des livres à la portée des con- 
seillers des cours supérieures. On observe le même silence dans les ajustements de l'ordonnance pour le contexte colonial. Nous ne savons toujours pas à quel ordonnance le procureur général fait référence.

${ }^{32}$ Délibération du Conseil de la Marine sur une requête du procureur général Mathieu-Benoit Collet, 17 mars 1717, BAC, Série $C^{11} \mathrm{~A}$, vol. 37, fol. 114-114v.

${ }^{33}$ Lettre de Philippe de Rigaud de Vaudreuil et Michel Bégon de la Picardière au Conseil de la Marine, 6 novembre 1717, BAC, Série $C^{11} \mathrm{~A}$, vol. 38, fol. 41. ${ }^{34}$ Idem.

${ }^{35}$ Prévu à l'origine comme un bâtiment autonome, le dépôt sera finalement installé dans les voûtes du palais, réaménagées à cet effet.

${ }^{36}$ Lettre de Charles de Beauharnois de la Boische et Gilles Hocquart à JeanFrédéric Phélypeaux, comte de Maurepas, 12 octobre 1731, BAC, Série $C^{11} \mathrm{~A}$, vol. 54 , fol. $139 \mathrm{v}$.

${ }^{37}$ Ce à quoi les autorités métropolitaines vont agréer, non sans en modérer l'ampleur. En octobre 1733, par exemple, le ministre de la Marine expédie 90 exemplaires de chacune des trois nouvelles déclarations du roi ayant trait aux actes notariés, alors qu'un premier document de travail parlait de 500 exemplaires de chacune des déclarations. Charles de Beauharnois de la Boische, Gilles Hocquart, Louis-Guillaume Verrier, Eustache Chartier de Lotbinière et François-Étienne Cugnet, « Procès-verbal de l'examen de trois projets de déclarations ", 6 octobre 1732, BAC, Série $\mathrm{C}^{11} \mathrm{~A}$, vol. 57 , fol. $264 \mathrm{v}$; lettre de Charles de Beauharnois de la Boische et Gilles Hocquart à Jean Frédéric Phélypeaux, comte de Maurepas, 3 octobre 1733, BAC, C ${ }^{11} \mathrm{~A}$, vol. 59, fol. 104.

${ }^{38}$ Pierre de Briquet, Code militaire; ou, Compilation des ordonnances des roys de France concernant les gens de guerre, Paris, Imprimerie royale, 1728.3 v. ; $17 \mathrm{~cm}$.

${ }^{39}$ Aux prises avec un important procès militaire, l'intendant, le gouverneur et un des écrivains du roi jugent en fait cette compilation de peu d'utilité dans les circonstances actuelles et ils réclament plutôt l'expédition d'un ouvrage comprenant des instructions précises sur la procédure militaire ainsi que des modèles de formules écrites. Ils n'ont au demeurant aucune idée d'un titre en particulier : « il n'est pas possible qu'il n'y en ayt quelques uns, que nous vous suplions Monseigneur de nous envoyer pour les distribuer dans les trois gouvernements »; Lettre de Charles de Beauharnois de la Boische et Gilles Hocquart à Jean-Frédéric Phélypeaux, comte de Maurepas, 23 octobre 1730, BAC, Série C ${ }^{11}$ A, vol. 52, fol. 124v-125.

40 Ibid., fol. 9v-10. 
${ }^{41}$ Lettre de Louis-Guillaume Verrier à Jean-Frédéric Phélypeaux, comte de Maurepas, 9 octobre 1733, BAC, Série $C^{11} \mathrm{~A}$, vol. 60, fol. 355v.

${ }^{42}$ Gabriel Argou, Institution au droit francois, Paris, Jean Mariette, 1730. 2 v.

${ }^{43}$ Lettre de Charles de Beauharnois de la Boische et Gilles Hocquart à JeanFrédéric Phélypeaux, comte de Maurepas, 1732, BAC, Série C ${ }^{11}$ A, vol. 57, fol. 10.

${ }^{44}$ En 1752, sept exemplaires de l'Ordonnance de la Marine sont recensés parmi les «munitions et ustencilles " pour les courses et voyages, travaux publics et services des magasins et bureaux du roi.

${ }^{45}$ Dans ce dernier cas, l'intendant spécifie qu'il souhaite celui : « en 4 . volumes in $f^{\circ}$ de la d.re Edition "; François Bigot « Etat des munitions Marchandises, et grand habillement quil est necessaire d'Envoyer de France, pour garnir les magasins du Roy a Quebec pour l'année mil sept cent cinquante. 1 », [1750], BAC, Série $C^{11} A$, vol. 93, fol. 343 et 345 .

${ }^{46}$ François Foucault, «Inventaire général des munitions et marchandises qui restent aujourd'hui dans les magasins du roi à Québec », 20 septembre 1738, BAC, Série $C^{11} A$, vol. 69, fol. 253v ; ibid., 1 octobre 1739, BAC, Série $C^{11} A$, vol. 72, fol. 12v ; ibid., 4 septembre 1741, BAC, Série $C^{11}$ A, vol. 75, fol. 291 ; ibid., 1 septembre 1742, BAC, Série $\mathrm{C}^{11} \mathrm{~A}$, vol. 78, fol. 78; Guillaume Estèbe, "Inventaire général des munitions, marchandises et vivres [...] dans les magasins de Québec ", 15 octobre 1743, BAC, Série $C^{11} \mathrm{~A}$, vol. 80, fol. 130 ; ibid., 20 octobre 1744, BAC, Série $C^{11} \mathrm{~A}$, vol. 82, fol. 146v-147 ; Ibid., 1 septembre 1749, BAC, Série C"A, vol. 93, fol. 332v.

${ }^{47}$ Il en va de même des « ornements de la chapelle qui est au Palais » et des "Instruments de Mathématiques remis au Professeur d'hydrographie », bref, de tous ces effets fournis par la couronne pour l'administration de la justice, la célébration du culte et l'enseignement collégial, mais qui ne se trouvent pas physiquement dans ses magasins.

${ }^{48}$ Un titre tronqué, réduit à sa plus simple expression, mais suffisamment distinctif pour être reconnu par ses contemporains.

49 Avec douze attributions d'auteurs pour vingt titres, les inventaires du garde-magasin du roi sont prodigues. Les inventaires, notamment ceux dressés lors du règlement d'une succession, sont généralement avares d'informations auctoriales. À y regarder de plus près, on remarque qu'une fois de plus, l'indication du nom de l'auteur n'a aucune finalité bibliographique. Elle sert uniquement à distinguer des ouvrages qui autrement seraient confondus et 
dont, par conséquent, la trace pourrait être perdue - ce qui réduirait la fonction conservatrice première de ce genre de répertoire. Ainsi en va-t-il de la " coutume de Paris par ferriere » et de la « coutume par Brodeau », de même que des « arrests de henry » et des « arrest de Loüet ». La recherche dans le catalogue des fonds anciens de la Bibliothèque nationale de France confirme d'ailleurs cette tendance puisque l'attribution des titres dont les auteurs ne sont pas indiqués à l'inventaire porte rarement à confusion. Autrement dit, les ouvrages auxquels le plumitif n'a accolé aucun nom d'auteur sont des ouvrages dont l'identification par le simple titre ne posait aucun problème à ses contemporains.

${ }^{50}$ Chacun des titres inventoriés par le garde-magasin ne compte qu'un exemplaire.

${ }^{51}$ Huit sont constitués d'un seul volume, sept, de deux volumes, trois, de trois volumes, un de quatre volumes et un de six volumes.

${ }^{52}$ Tous des in-folio, à l'exception d'un in-douze.

${ }^{53}$ Inventaire après décès des biens de Louis-Guillaume Verrier, 10 janvier 1759, ANQQ, greffe Jean-Baptiste Decharnay.

${ }^{54}$ « Balance et Recette... pour les magasins du Roy, l'année 1749 », BAC, Série $\mathrm{C}^{11} \mathrm{~B}$, vol. 28, fol. 202-265.

${ }^{55} \mathrm{Ne}$ retenons que deux exemples au passage : Henri-Jean Martin, Histoire et pouvoirs de l'écrit, Paris, Perrin, 1988, 518 p. ; Marc Baratin et Christian Jacob, dir, Le pouvoir des bibliothèques. La mémoire des livres en Occident, Paris, Albin Michel, 1996, $338 \mathrm{p}$.

${ }^{56}$ Le signe « + » indique des ouvrages qui ont été commandés par les administrateurs locaux ou qui leur ont été expédiés et qui sont identifiés nommément dans la correspondance officielle. Ils ne se trouvaient toutefois pas à l'inventaire du garde-magasin.

${ }^{57}$ Généralement, nous indiquons ici le lieu de localisation d'exemplaires qui semblent correspondre à la même édition que celle du catalogue. Puisqu'ils n'ont pas été vus par nous, il est impossible pour le moment de dire s'il s'en trouve parmi eux qui ont effectivement appartenu à la collection du Conseil supérieur de Québec. Cette information pourra guider cette recherche ultérieure, mais surtout, elle permet aux chercheurs intéressés à l'histoire socio juridique de la Nouvelle-France de retrouver rapidement les textes de référence. Notre principal outil a été le catalogue en-ligne Amicus de Bibliothèque 
et Archives Canada. Quand aucune référence n'y était disponible, nous avons utilisé le catalogue en-ligne Opale-Plus de la Bibliothèque nationale de France.

${ }^{58}$ Les « mentions documentaires » font référence à l'information délivrée par les inventaires du garde-magasin, de la note d'emprunts de Verrier, ainsi que de son inventaire après décès.

${ }^{59}$ Il existe plusieurs éditions de ce dictionnaire publié pour la première fois, à titre posthume, en 1690. Nous avons retenu cette édition en raison des propos de l'intendant Bigot qui spécifiait dans sa commande obtenir la dernière édition en quatre volumes in-folio. Au catalogue de la Bibliothèque nationale de France et à celui de Bibliothèque et Archives du Canada, l'édition hollandaise est la seule qui correspond aux spécifications de l'administrateur. La veuve Delaulne, à Paris, publie une autre édition en 1732, mais elle comporte 5 volumes.

${ }^{61}$ La première édition date 1578. Comme l'ouvrage n'a pas été emprunté par Verrier, nous n'avons aucune indication précise quant à l'édition conservée à Québec. Nous avons choisi d'inscrire ici la référence de la dernière édition augmentée de ce livre dont on ne trouve pas trace au catalogue Amicus.

${ }^{61} \mathrm{~L}$ 'information laconique permet difficilement l'identification de cet ouvrage. Nous savons cependant qu'il comporte six volumes ce qui nous laisse à penser qu'il s'agit fort probablement du Corpus juris civilis qui sert de fondement au droit civil français. Il s'agit d'une compilation réalisée sous le règne de Justinien, vaste recueil de droit romain qui recouvre un triple domaine : législatif (Code et Novelles), doctrinal (Digeste) et pédagogique (Institutes). Claude de Ferrière en a d'ailleurs fait une édition française. Il est tentant d'y voir aussi une référence aux Loix civiles de Jean Domat. Mais rien ne nous permet de trancher pour le moment.

${ }^{62}$ Nous avons été incapable d'identifier ce document jusqu'à ce jour. 